\section{Enzymatic microRNA detection in microtiter plates with DNA dendrimers}

\author{
Johanna R. Mora and Robert C. Getts \\ Genisphere, Inc., Hatfield, PA, USA
}

BioTechniques 41:420-424 (October 2006)

doi 10.2144/000112270
microRNAs (miRNAs) are small noncoding RNA oligonucleotides (approximately 22 nucleotides) that repress gene expression in two ways: (i) pair exactly to target regions in messenger RNA and cause its degradation or (ii) pair partially to target regions and inhibit translation of the message (1). Recent studies (2-5) indicate that miRNAs play an important role in cellular development and disease. This has prompted many scientists to study miRNAs in different species and at different developing stages, as well as to compare normal and carcinogenic samples. Northern blot analyses $(6,7)$ are considered the gold standard for miRNA detection, but the method does not allow highthroughput sample analysis. Several research groups have developed microRNA-specific oligonucleotide microarrays (8-10) with comparable capabilities and higher sensitivity and throughput than Northern blot analyses. Unfortunately, the equipment required for microarray analysis is expensive and therefore not available in every research or diagnosis laboratory. We have developed a fast and inexpensive method for the detection of miRNA in microtiter plates using horseradish peroxidase (HRP)-conjugated DNA dendrimers as detection molecules for signal amplification.

DNA dendrimers are three-dimensional (3-D) branched structures of DNA (11) with multiple arms (singlestranded DNA) that can be functionalized or labeled with a diverse range of molecules such as fluorophores, biotins, antibodies, and enzymes. DNA dendrimers are ideally suited for signal amplification and have been commercially adapted for the amplification of fluorescent signal of microarrays
(12). Here, we present a new application for the use of conjugated DNA dendrimers.

To enable detection of miRNAs we spotted probes designed by Goff and coworkers (10) onto microtiter plates (sequences are provided in Supplementary Table S1 available online at www.BioTechniques.com). Low molecular weight (LMW) rat RNA and control oligonucleotides were labeled at the $3^{\prime}$ end with a 31 base oligonucleotide complementary to the outer arms of the dendrimer (Supplementary Figure S1). Labeled control oligonucleotides and samples were diluted in miRNA hybridization buffer (see supplementary material), and added to the wells of the spotted plates. After washing to remove unbound miRNAs, two types of dendrimers were evaluated as detection molecules: $(i)$ biotinylated dendrimers for detection with streptavidin horseradish peroxidase (SA-HRP) and (ii) HRP dendrimer conjugates requiring no additional steps except washing before detection. Color was developed by addition of tetramethylbenzidine
(TMB; HRP substrate). To best qualify the significance of the results, two types of negative control experiments were conducted to determine the background contribution of all processing steps and components: no spotted oligonucleotide, and no input miRNA sample. The background values ranged from 0.041 to 0.061 and 0.045 to 0.066 , respectively, depending on the assay stringency, and are typical for this type of enzymatic assay. A detailed description of reagents and methods can be found in the supplementary material.

To confirm that the observed binding was specific, we analyzed the tissue specificity of LMW total rat RNA from three different tissues: heart, liver, and brain. We confirmed that miR-1 was predominantly present in heart tissue, miR-122 in liver, and miR-124a in brain. Low levels of miR-1 have been previously observed to be present in liver and brain (13). However, like in Northern blot analysis, this method failed to identify miR-1 in either tissue (Figure 1).

To determine how well the method would discriminate mutations of the same miRNA, we designed two miR-124a oligonucleotides, one with a mutation at base 10 and a second with mutations at both base 4 and 16. We modified the hybridization stringency by titrating the formamide concentration during the miRNA control oligonucleotide hybridization. Using hybridization buffer with $60 \%$ formamide, the signal from the singlenucleotide mutant was only $10 \%$ the signal from the wild-type sequence,

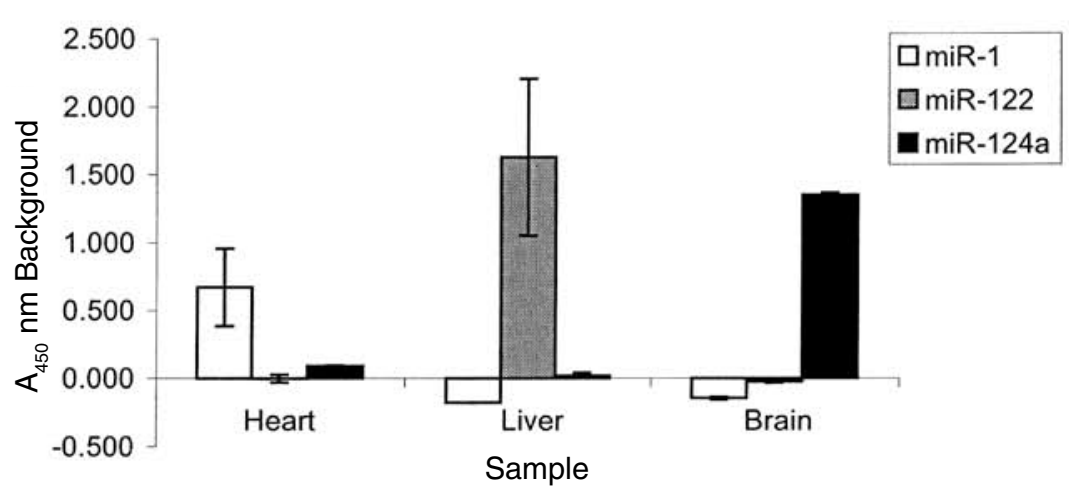

Figure 1. Tissue specificity in microRNA (miRNA) samples. Detection of $70 \mathrm{ng}$ low molecular weight (LMW) RNA-enriched samples on a microtiter plate coated with miR-124a, miR-122, and miR-1 probes. Sixty nanograms dendrimer (960 biotins) per well were used for the detection. 


\section{Benchmarks}

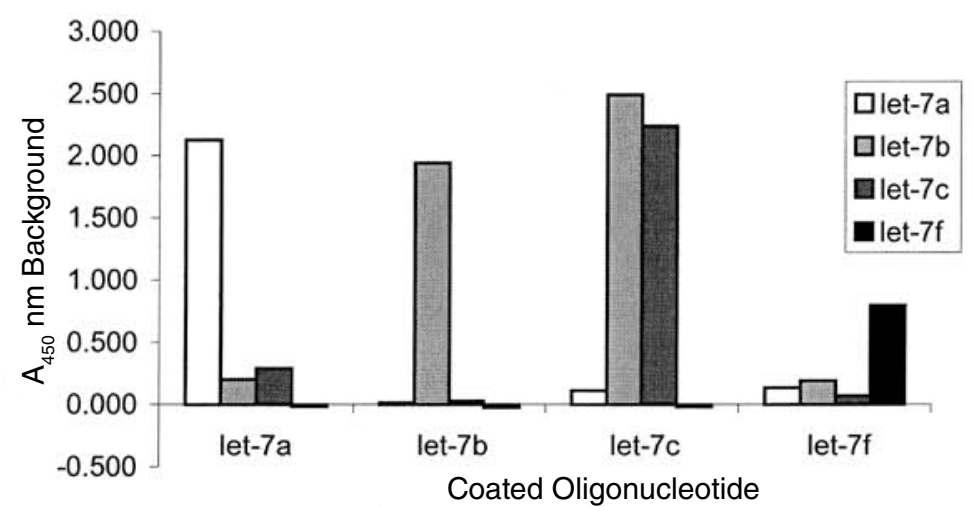

Figure 2. Optimized detection of let-7 control oligonucleotides. The sample hybridization buffer consisted of $1.125 \mathrm{M}$ tetramethylammonium chloride (TMAC), $1.5 \mathrm{mM}$ EDTA, $18.75 \mathrm{mM}$ Tris- $\mathrm{HCl}, \mathrm{pH}$ 8.0 , and $40 \%$ formamide. The hybridization was carried out at $25^{\circ} \mathrm{C}$, and detection was done with $30 \mathrm{ng}$ two-layer horseradish peroxidase (HRP) dendrimer conjugate (30 HRPs/dendrimer).

and the signal from the double mutant was $<1 \%$ (see Supplementary Figure S2). Comparing these observations to those reported on microarrays by Goff and colleagues (10) suggests that microtiter plate assays are better able to discriminate miRNAs differing by only 1 or 2 bases. We also found that the use of dextran sulfate as a volume excluder in the miRNA hybridization buffer at a concentration of $2 \%-3 \%$ increased the output signal, presumably by favoring the hybridization of sample to target probe.

The optimal mass of dendrimer/ well was determined to be $60 \mathrm{ng} / \mathrm{well}$. HRP-labeled dendrimers (30 HRPs/ dendrimer) gave a signal over noise ratio $(\mathrm{S} / \mathrm{N})$ about 5 -fold greater than biotinylated dendrimers (960 biotins/ dendrimer) chased with SA-HRP. This could be explained by the inability of the larger (four-layer) biotinylated dendrimer to bind as many targets as the smaller (two-layer) HRP-labeled dendrimer, due to steric hindrance. Alternatively, having the HRP directly attached to minimize extra steps may also be advantageous. The output signal achieved with the different dendrimer conjugates is summarized in Supplementary Table S2.

The smallest discernible analytical signal was determined according to the International Union of Pure and Applied Chemistry's (IUPAC's) definition (14). For the control oligonucleotides (Supplementary Figure S3) under the more stringent conditions, it was found to be 0.048 absorbance units, which corresponds to 4.9 pg oligonucleotides/ well. The same determination was done using LMW total RNA from rat liver and brain, and the smallest discernible analytical signal corresponds to 2.15 and $0.909 \mathrm{ng}$, respectively, using the Microcon ${ }^{\circledR}$ YM-100 microconcentrator-enriched RNA/well (Millipore, Billerica, MA, USA).

The discrimination among let-7 orthologs required the use of tetramethylammonium chloride (TMAC), and N-lauroyl-sarcosine in the miRNA hybridization buffer. TMAC is known to eliminate the preferential melting of AT versus GC base pairs and can be used to obtain hybridization of only exact matches (15-17). Figure 2 shows the final discrimination results obtained using hybridization buffer B (1.125 M TMAC, $1.5 \mathrm{mM}$ EDTA, $18.75 \mathrm{mM}$ Tris- $\mathrm{HCl}, \mathrm{pH} 8.0$, and $40 \%$ formamide) at $25^{\circ} \mathrm{C}$. Under those conditions most let-7 oligonucleotides can be discriminated, except for let-7b, which bound to both $l e t-7 b$ and $l e t-7 c$ spotted probes, presumably due to the position of the mutation (toward one end of the probe instead of in the middle where it would cause a greater disruption in the hybridization). The optimization of TMAC concentration in the hybridization buffer for detection of let-7c shown in Supplementary Figure S4.

While this method is not ideally suited for miRNA profiling, we studied let-7 series profile in brain and liver (Supplementary Figure S5). The distribution of the studied let-7 miRNAs in brain, as well as the down-regulation of the let-7 series in liver relative to brain, agrees with previously reported results $(2,13,18)$.

This method has several advantages for miRNA analysis over microarrays: (i) it uses shorter experimental time; (ii) it is less expensive; and (iii) it has high sample throughput for studying the expression of the same miRNA in many different samples. The disadvantages are: $(i)$ a given experiment can only investigate as many sequences as the number of wells analyzed and (ii) in most cases, each well requires approximately 3 ng labeled LMW RNA to generate $\mathrm{S} / \mathrm{N}>3$. However, the study of some miRNAs, such as let-7f might require more than $30 \mathrm{ng} / \mathrm{well}$. This may be achieved by amplifying the miRNA using recently described methods (19). Optimization of sample hybridization buffer (concentration of formamide, TMAC, dextran sulfate, and surfactants) allowed, in some cases, discrimination of single-base mutations among miRNAs. Salt concentration, dextran sulfate percentage, and overall conjugate concentration in the dendrimer hybridization buffer were determined to be important parameters in the optimization of UltraAmp ${ }^{\mathrm{TM}}$ reagent (Genisphere, Hatfield, PA, USA) hybridizing to target. As would be expected, the UltraAmp Biotin (960) gave greater signal amplification than UltraAmp Biotin (45) reagents. However, dendrimer directly labeled with HRP outperformed any biotinylated dendrimer detected with SA-HRP.

\section{ACKNOWLEDGMENTS}

We are grateful to Ronald Hart and Loyal Goff for the probe design and miRNA profiling results.

\section{COMPETING INTERESTS STATEMENT}

J.R.M. and R.G. work at Genisphere, the company that makes the dendrimers used for the detection of miRNAs. Genisphere could possibly benefit from the publication of this paper. 


\section{Benchmarks}

\section{REFERENCES}

1.Lim, L.P., N.C. Lau, P. Garett-Engele, A. Grimson, J.M. Schelter, J. Castle, D.P. Bartel, P.S. Linsley, and J.M. Johnson. 2005. Microarray analysis shows that some microRNAs downregulate large numbers of target mRNAs. Nature 433:769-773.

2. Miska, E.A., E. Alvarez-Saavedra, M. Townsend, A. Yoshii, N. Sestan, P. Rakic, M. Constantine-Paton, and H.R. Hortovitz. 2004. Microarray analysis of microRNA expression in the developing mammalian brain. Genome Biol. 5:R68.1-R68.13.

3. Krichevsky, A.M., K.S. King, C.P. Donahue, K. Khrapko, and K.S. Kosik. 2003. A microRNA array reveals extensive regulation of microRNAs during brain development. RNA 9:1274-1281.

4. Esau, C., X. Kang, E. Peralta, E. Hanson, E.G. Marcusson, L.V. Ravichandran, Y. Sun, S. Koo, et al. 2004. MicroRNA-143 regulates adipocyte differentiation. J. Biol. Chem. 279:52361-52365.

5. Michael, M.Z., S.M. O'Connor, N.G. van Holst Pellekaan, G.P. Young, and R J. James. 2003. Reduced accumulation of specific microRNAs in colorectal neoplasia. Mol. Cancer Res. 1:882-891.

6. Sempere, L.F., S. Freemantle, I. PithaRowe, E. Moss, E. Dmitrovsky, and V. Ambros. 2004. Expression profiling of mammalian microRNAs uncovers a subset of brain-expressed microRNAs with possible roles in murine and human neuronal differentiation. Genome Biol. 5:R13.

7. Ambros, V., R.C. Lee, A. Lavanway, P.T. Williams, and D. Jewell. 2003. MicroRNAs and other tiny endogenous RNAs in C. elegans. Curr. Biol. 13:807-818.

8. Barad, O., E. Meiri, A. Avniel, R. Aharonov, A. Bazilai, I. Bentwich, U. Einav, S. Gilad, et al. 2004. MicroRNA expression detected by oligonucleotide microarrays: System establishment and expression profiling in human tissues. Genome Res. 14:2486-2494.

9. Nelson, P.T., D.A. Baldwin, L.M. Scearce, J.C. Oberholtzer, J.W. Tobias, and Z. Mourelatos. 2004. Microarray-based, highthroughput gene expression profiling of microRNAs. Nat. Methods. 1:155-161.

10. Goff, L.A., M. Yang, J. Bowers, R.C. Getts, R.W. Padgett, and R.P. Hart. 2005. Rational probe optimization and enhanced detection strategy for microRNAs using microarrays. RNA Biol. 2:e9-e16.

11. Nilsen, T.W., J. Grayzel, and W. Prensky. 1997. Dendritic nucleic acid structures. J. Theor. Biol. 187:273-284.

12. Stears, R.L., R.C. Getts, and S.R. Gullans. 2000. A novel, sensitive detection system for high-density microarray using dendrimer technology. Physiol. Genomics 3:93-99.

13. Lagos-Quintana, M., R. Rauhut, A. Yaicin, J. Meyer, W. Lendeckel, and T. Tuschl. 2002. Identification of tissue-specific microRNAs from mouse. Curr. Biol. 12:735-739.

14. Long, G.L. and J.D. Winefordner. 1983. Limit of detection a closer look at the IUPAC definition. Anal. Chem. 55:712A-724A.

15. Wood, W.I., J. Gitschier, L.A. Lasky, and R.M. Lawn. 1985. Base composition-inde- pendent hybridization in tetramethylammonium chloride: a method for oligonucleotide screening of highly complex gene libraries. Proc. Natl. Acad. Sci. USA 82:1585-1588.

16. Connors, T.D., T.C. Burn, T. VanRaay, G.G. Germino, K.W. Klinger, and G.M. Laudes. 1997. Evaluation of DNA sequencing ambiguities using tetramethylammonium chloride hybridization conditions. BioTechniques 22:1088-1090.

17. Borucki, M.K., J. Reynolds, D.R. Call, T. Ward, B. Page, and J. Kadushin. 2005. Suspension microarrays for direct and high throughput subtyping of Listeria monocytes from genomic DNA. J. Clin. Microbiol. 43:3255-3259.

18. Thomson, J.M., J. Parker, C.M. Perou, and S.M. Hammond. 2004. A custom microarray platform for analysis of microRNA gene expression. Nat. Methods 1:47-53.

19. Mattie, M.D., C.C. Benz, J. Bowers, K. Sensinger, L. Wong, G.K. Scott, V. Fedele, D.G. Ginzinger, et al. 2006. Optimized highthroughput microRNA expression profiling provides novel biomarker assessment of clinical prostate and breast cancer biopsies. Mol. Cancer 5:24.

Received 14 July 2006; accepted 2 August 2006.

Address correspondence to Robert C. Getts, 2801 Sterling Drive, Hatfield, PA 19440, USA.e-mail:Bob_Getts@datascope.com

To purchase reprints of this article, contact: Reprints@BioTechniques.com 